\title{
Hepatocellular carcinoma presenting as right supraclavicular lymphadenopathy
}

\author{
Douglas Thorburn MD MRCP, Faisal M Sanai MD SBG, Cameron N Ghent MD FRCPC
}

D Thorburn, FM Sanai, CN Chent. Hepatocellular carcinoma presenting as right supraclavicular lymphadenopathy. Can J Gastroenterol 2003;17(10):605-606.

Two patients, one with previously undiagnosed liver disease, presenting with right supraclavicular lymphadenopathy were subsequently diagnosed with hepatocellular carcinoma. This presentation has only been previously described once, and the mechanism of this unusual presentation is discussed.

Key Words: Hepatocellular carcinoma; Right supraclavicular lymphadenopathy

\section{Hépatocarcinome sous forme d'adénopathie supraclaviculaire droite}

\begin{abstract}
Deux patients présentant une adénopathie supraclaviculaire droite, dont l'un atteint d'une maladie hépatique auparavant non diagnostiquée,ont ensuite chez qui on a ensuite découvert un hépatocarcinome; cette présentation inhabituelle n'a été décrite qu'une fois auparavant, et son mécanisme est abordé.
\end{abstract}

\section{CASE PRESENTATIONS}

Patient 1

A 67-year-old man, with no significant past medical history, presented in August 2002 with a short history of malaise, weight loss, intermittent confusion, ankle edema and jaundice. For many years he had consumed up to a bottle of spirits a day, but had abstained completely from alcohol for the six years prior to this presentation. On examination he was jaundiced, with multiple spider nevi, asterixis and a $2 \mathrm{~cm}$ right supraclavicular lymph node. Abdominal examination revealed the left lobe of liver extending $15 \mathrm{~cm}$ below the subcostal margin. Abdominal ultrasonography revealed an irregular lobulated liver, ascites and splenomegaly consistent with cirrhosis. There was an irregular $9 \times 8 \times 5 \mathrm{~cm}$ mass in the left lobe of liver, three lymph nodes up to $4 \mathrm{~cm}$ in diameter in the porta hepatis and a portal vein thrombosis. In view of persistent coagulopathy despite vitamin K, a biopsy of the intra-abdominal mass was not attempted. Fine needle aspiration of the right supraclavicular node revealed features consistent with hepatocellular carcinoma (HCC). Subsequently, the serum alpha-fetoprotein level was confirmed to be elevated at $198 \mathrm{UG} / \mathrm{L}$ (normal less than $10 \mathrm{UG} / \mathrm{L})$.

\section{Patient 2}

In August 1999, a 72-year-old woman with known primary biliary cirrhosis $(\mathrm{PBC})$ and autoimmune hepatitis type 1 overlap syndrome presented with a $5 \mathrm{~cm}$ firm, nontender right supraclavicular lymphadenopathy. PBC had been diagnosed in 1983 on the basis of cholestatic liver function tests, immunoglobulin (Ig) M elevation, positive antimitochondrial antibody and consistent histology with portal based granulomas and stage 3 fibrosis. In 1985, she became jaundiced with markedly elevated transaminases, positive antinuclear and antismooth muscle antibodies, elevated IgG and marked interface hepatitis on liver biopsy. She was treated with azathioprine and prednisone with normalization of transaminases and remained well without hepatic decompensation until this presentation. Chest $\mathrm{x}$ ray and bronchoscopy were normal. Upper gastrointestinal endoscopy revealed only grade 2 esophageal varices. A computed tomography scan of the chest and abdomen revealed multiple heterogeneous masses of up to $8 \mathrm{~cm}$ throughout the liver, lymphadenopathy at the porta hepatis and anterior pericardial, precarinal and anterior mediastinal lymphadenopathy. Fine needle aspiration of the right supraclavicular lymph node confirmed features consistent with HCC. Alpha-fetoprotein was greater than $3000 \mathrm{UG} / \mathrm{L}$. She was offered palliative chemotherapy; however, her condition rapidly deteriorated and she died 11 months after the lymphadenopathy was first detected.

\section{DISCUSSION}

In autopsy studies of patients with HCC, hematogenous dissemination following vascular invasion is extremely common. Lymph node metastases, usually confined to the abdominal or thoracic cavity, are less common and were found in approximately $30 \%$ of cases in a large postmortem series (1). Lymphatic spread can be explained by the finding that even relatively small HCCs contain lymphatic vessels that drain to local lymph nodes (2).

Distant sites of lymphatic metastases from HCC are uncommon, but have been reported in postmortem series and a few case reports. In particular, left supraclavicular lymphadenopa-

Liver Unit, London Health Sciences Centre, University of Western Ontario, London, Ontario

Correspondence: Dr Douglas Thorburn, Multiple Organ Transplant Unit, London Health Sciences Centre, 339 Windermere Road, London, Ontario N6A 5A5. Telephone 519-663-3406, fax 519-663-4858, e-mail douglasthorburn@hotmail.com

Received for publication March 17, 2003. Accepted July 112003 
thy (Virchow-Troisier's node) has been described in three cases (3-5), with a single previous report of HCC presenting as right supraclavicular lymphadenopathy (6). Metastasis to the left supraclavicular nodes is the result of lymph draining from most of the liver to nodes in the porta hepatis and thereafter to celic nodes and the left thoracic duct, as is observed in gastric carcinoma (5). In addition, some of the capsular lymphatics draining from the right lobe of liver communicate with the diaphragmatic lymphatics and thereafter the mediastinal and paratracheal lymph nodes (7). In approximately 20\% of people, these unite with the right subclavian and jugular lymphatic trunks to form a right lymphatic duct which drains into the junction of the right internal jugular and subclavian veins. This is the most likely mechanism of right supraclavicular lymphadenopathy in our patients. Interestingly, the first patient that we report had a large lesion within the left lobe of liver, an area not expected to drain in the manner described; however, it is recognized that in liver cirrhosis the lymphatic drainage of the liver can be altered due to increased lymph production and obstruction of lymphatic vessels by fibrosis (7).

\section{CONCLUSIONS}

$\mathrm{HCC}$ is recognized to present in unusual ways in patients with undiagnosed chronic liver disease, as was the case with the first patient. This diagnosis should be considered in patients pre- senting with right supraclavicular lymphadenopathy regardless of a prior diagnosis of cirrhosis and can be easily confirmed by abdominal imaging, alpha-fetoprotein assay and fine needle aspiration. Unfortunately, as observed with these cases, distant lymphatic spread usually reflects advanced local disease and is associated with an extremely poor prognosis.

\section{REFERENCES}

1. Yoshioka M, Yamamoto M, Fujii H, et al. Characterisitics of lymph node metastases of hepatocellular carcinoma comparing to cholangiocellular carcinoma - a statistical analysis of annual of the pathological autopsy cases in Japan from 1972 to 1981. Acta Hepatol Jpn 1985;26:1034-9.

2. Saitsu H, Kojiro M, Taniwaki S, et al. Percutaneous injection of adriamycin-lipiodol emulsion into hepatocellular carcinoma tumor nodules demonstrates lymph vessels connecting to the tumor and the regional lymph nodes. Kurume Med J 1987;34:119-24.

3. Benner EJ, Labby DH. Hepatoma: Clinical experiences with a frequently bizarre tumour. Ann Intern Med 1961;54:620-35.

4. Schwarz KO, Schwartz IS, Marchevsky A. Virchow-Troisier's lymph node as the presenting sign of hepatocellular carcinoma. Mt Sinai J Med 1982;49:59-62.

5. Kew MC. Virchow-Troisier's lymph node in hepatocellular carcinoma. J Clin Gastroenterol 1991;13:217-9.

6. Lau KK, Wong KW, Ho WC, et al. Hepatocellular carcinoma presenting with right supraclavicular lymph node metastasis and superior mediastinal syndrome. Liver 2000;20:184-5.

7. Magari S. Hepatic lymphatic system: Structure and function. J Gastroenterol Hepatol 1990;5:82-93. 


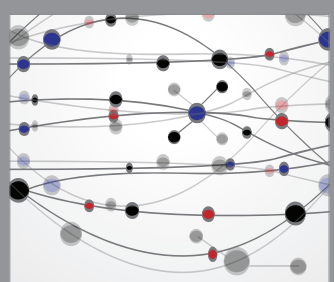

The Scientific World Journal
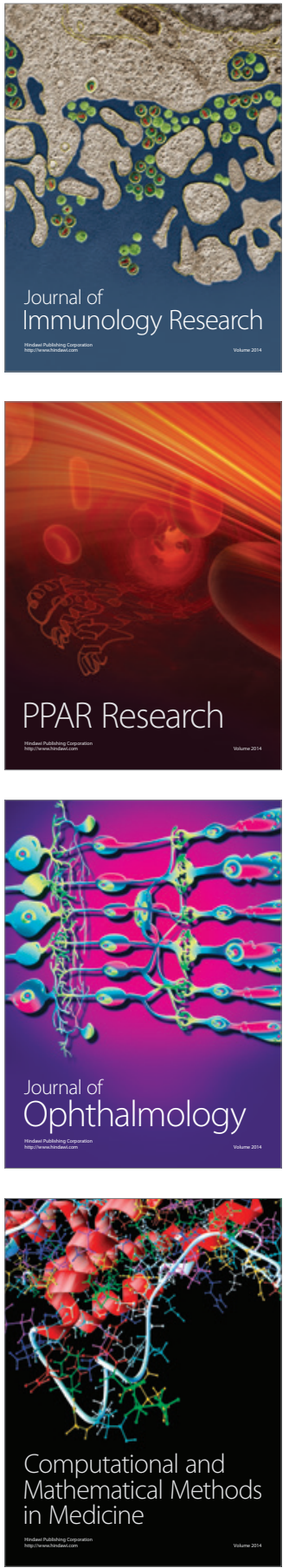

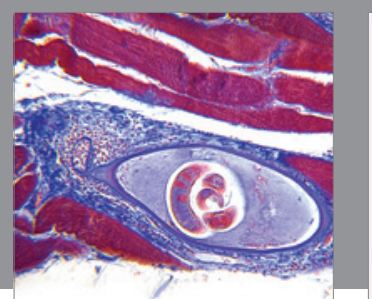

Gastroenterology Research and Practice

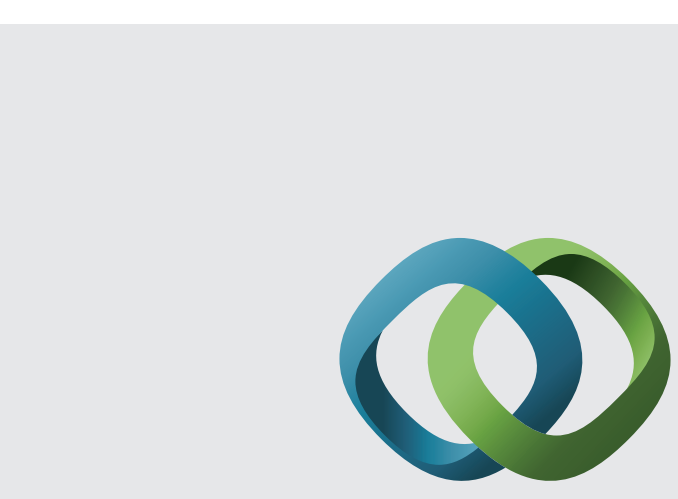

\section{Hindawi}

Submit your manuscripts at

http://www.hindawi.com
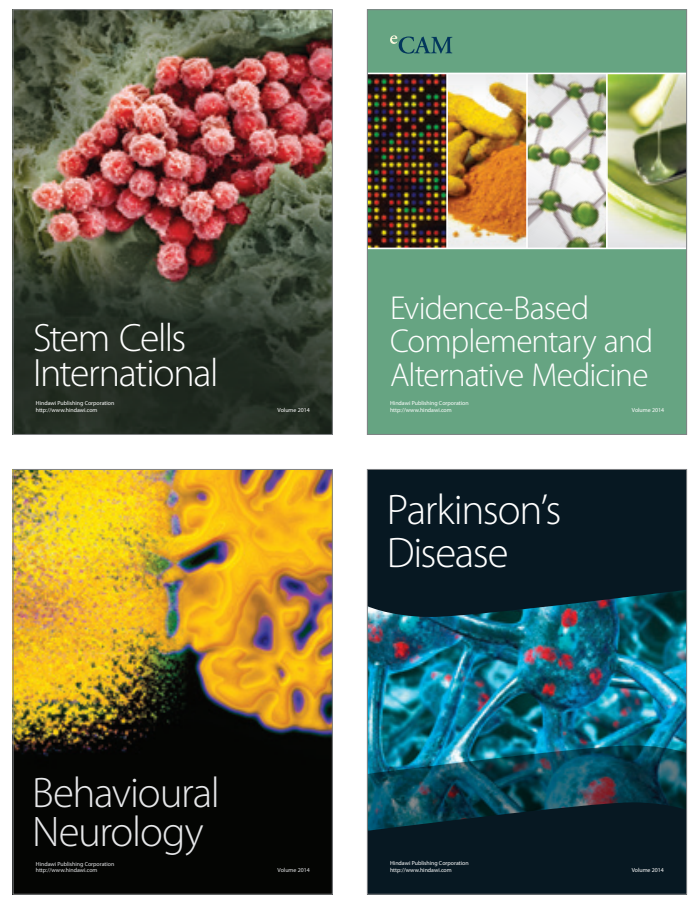
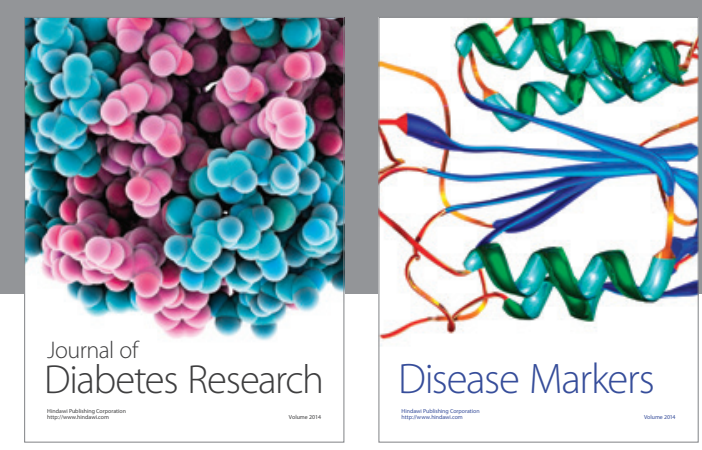

Disease Markers
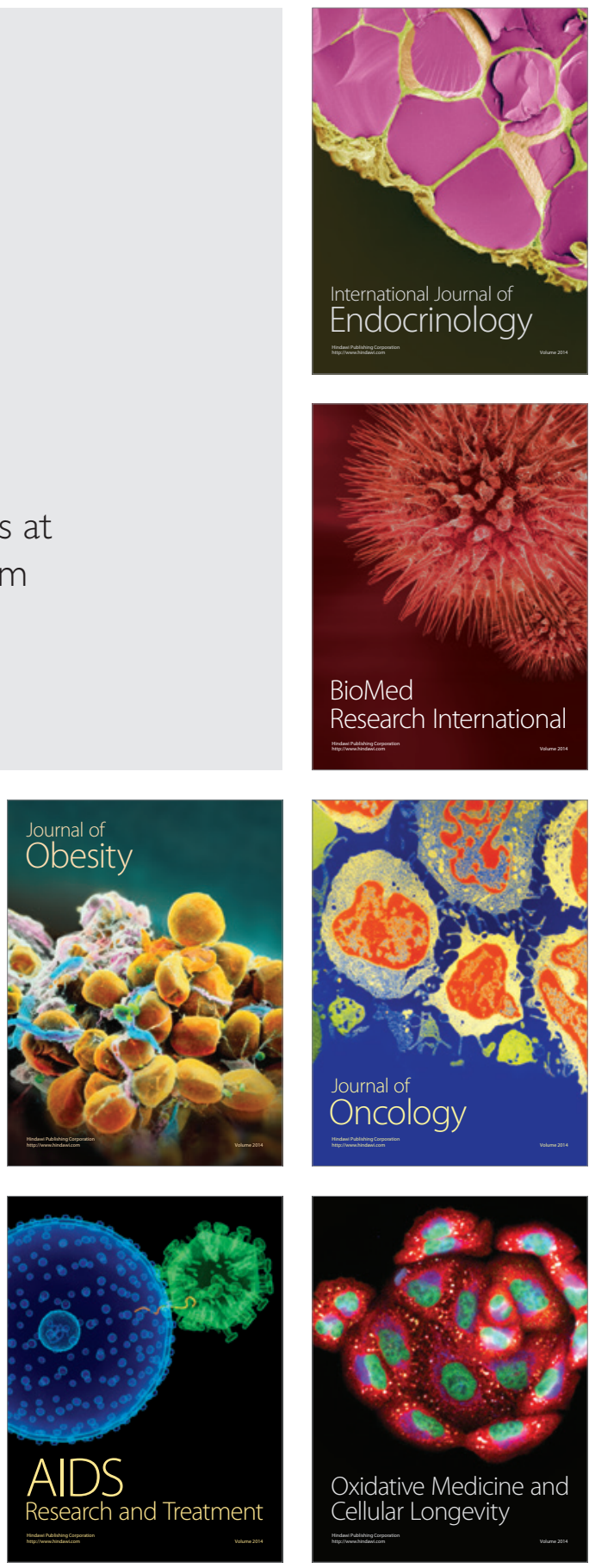